\title{
Application and Mechanism of Malonic Acid as a Green Alternative for Protein-Crosslinking
}

\author{
Donald Szlosek, Douglas Currie \\ Department of Biological Sciences, University of Southern Maine, Portland, Maine \\ Email: Donald.szlosek@maine.edu
}

Received 14 April 2016; accepted 21 May 2016; published 24 May 2016

Copyright (C) 2016 by authors and Scientific Research Publishing Inc.

This work is licensed under the Creative Commons Attribution International License (CC BY). http://creativecommons.org/licenses/by/4.0/

(c) (i) Open Access

\begin{abstract}
Formaldehyde fixation is the main method for crosslinking cellular proteins prior to their usage in immunocytochemistry. In order to create these links, formaldehyde undergoes a Mannich reaction in which the formaldehyde forms a methylene bridge between the aminogroup of two amino acids. Crosslinking increases protein stability allowing for more accurate preservation of in vivo conformations which in turn increases binding affinity of fluorochrome conjugated antibodies for fluorescent imaging. Formaldehyde is also a known carcinogen as classified by the National Cancer Institute. Malonic acid, a green, plant-based, water-soluble, and relatively inexpensive polycarboxylic acid has been shown to undergo crosslinking of proteins through an unknown mechanism. To test whether malonic acid can crosslink proteins within cells we fixed SH-5YSY cells with either malonic acid or formaldehyde and then stained with a fluorochrome conjugated antibody for the cytoskeletal protein $\alpha$-tubulin. The cells were then imaged 72 hours after fixation. We observed a non-significant difference in the fluorescence of immunostained SH-5YSY cells fixed with malonic acid as compared to paraformaldehyde ( $p$-value $=0.2469$, ANOVA). In addition, we have created a theoretical mechanism showing malonic acid forming a propyl bridge for crosslinking proteins in a similar mechanism to that of formaldehyde. Here, we show that malonic acid is able to fix cells and retain fluorescence just as well as paraformaldehyde up to 72 hours after fixation and present several possible mechanisms for this chemical process.
\end{abstract}

\section{Keywords}

Green Chemistry, Protein Fixation, Protein Crosslinking, Cellular Fixation, Formaldehyde Fixation, Fixation, Malonic Acid 


\section{Introduction}

\subsection{Cellular Fixation}

Cellular fixation is an important step for the use of processing monolayers of cells during immunocytochemistry. Fixation helps preserve the overall cytoskeletal use of additional processing. Furthermore, fixation has been shown to essentially freeze proteins and other biological molecules in their spatial locations within the cell [1] [2].

For over 100 years formaldehyde has been used as one of the most common histological and cellular tissue fixatives [3] [4]. Formaldehyde reacts with the primary amines to crosslink proteins. Formaldehyde can combine with nitrogen and some other atoms of proteins or with two such atoms if they are very close together, forming a crosslink $-\mathrm{CH}_{2}$ - called a methylene bridge. Studies of the fixation of collagen have shown that the most frequent type of crosslink formed by formaldehyde in collagen is between the nitrogen atom at the end of the side-chain of lysine and the nitrogen atom of a peptide linkage [5].

Though this inherent chemical reactivity is useful for protein crosslinking, formaldehyde can be detrimental to human health and has been found to be carcinogenic by the National Toxicology Program [6]. Due to its toxicity, exposure to formaldehyde should be limited and a non-carcinogenic alternative is desired.

Malonic acid (propanedioic acid) is a dicarboxylic acid normally consisting of white crystals and is generally used as a precursor molecule for polyesters and alkyd resins [7]. Malonic acid is naturally found in high concentrations in beets and was first synthesized through the oxidation of malic acid extracted from rhubarb plants [8]. Malonic acid has been used to develop three-dimensional scaffolds of chitosan and collagen for biomedical applications through covalent amide/imine linkages [9]. In this study we compare the use of malonic acid with formaldehyde as an appropriate fixative for the immunostaining of in vitro cell cultures. In addition, we provide several theoretical mechanisms as to how malonic acid forms a protein crosslink.

\subsection{Formaldehyde Fixation}

The canonical protein crosslinking mechanism using formaldehyde is initiated by the hydration of formaldehyde into methylene glycol which can react together to form larger polymers. The neutral $\mathrm{pH}$ of tissues causes the methylene glycol to dehydrate into carbonyl formaldehyde. Both the hydrated and unhydrated forms of formaldehyde fix the tissue.

Having a carbonyl group on formaldehyde causes a strong dipole moment to be formed between the partially negative oxygen and the partially positive carbon. This dipole is what gives formaldehyde-its electrophilic properties and allows it to readily react with biological macromolecules. It has been observed that when placed in a collagen solution, formaldehyde frequently reacts to form a linkage between the nitrogen atom at the end of the side-chain of lysine and the nitrogen atom of a peptide linkage and the number of such crosslinks increases with time.

The crosslinking of proteins by formaldehyde is described as follows: 1) Formaldehyde, in aqueous solution, becomes hydrated to form a glycol intermediate (hydrated formaldehyde) called methylene glycol. 2) Methylene glycol reacts with a primary amine through the nucleophilic addition of the carbonyl and dehydration of the Schiff base. 3) The Schiff base is an electrophile which reacts in the second step in an electrophilic addition with a compound containing an acidic proton. 4) The subsequent crosslinking occurs by formation of a crosslink of $-\mathrm{CH}_{2}$ - known as a methylene bridge (Figure 1).

\section{Materials and Methods}

\subsection{Cell Culture}

SH-5YSY cells were purchased from the American Type Culture Collection (ATCC, Manassas, VA) and maintained in a 1:1 mixture of EMEM and FK12 media supplemented with 10\% fetal bovine serum (ATCC), 100 $\mathrm{U} / \mathrm{mL}$ penicillin and $100 \mathrm{ug} / \mathrm{mL}$ streptomycin (HyClone, Logan UT). All components and complete medium were warmed prior to use. Cells were grown in $5 \mathrm{~mL}$ medium in T-25 plug-seal tissue culture flasks (Corning, Corning, NY) kept at $37^{\circ} \mathrm{C}$ and $5 \% \mathrm{CO}_{2}$ in a Nuaire (Plymouth, MN) NU-4750 incubator. For harvesting, medium was removed from the flasks and the cells rinsed with $\mathrm{Ca}^{2+} / \mathrm{Mg}^{2+}$-free Dulbecco's phosphate-buffered sa-

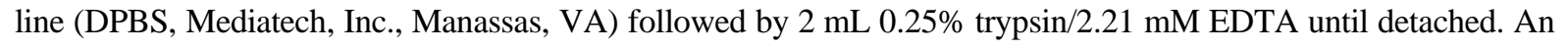




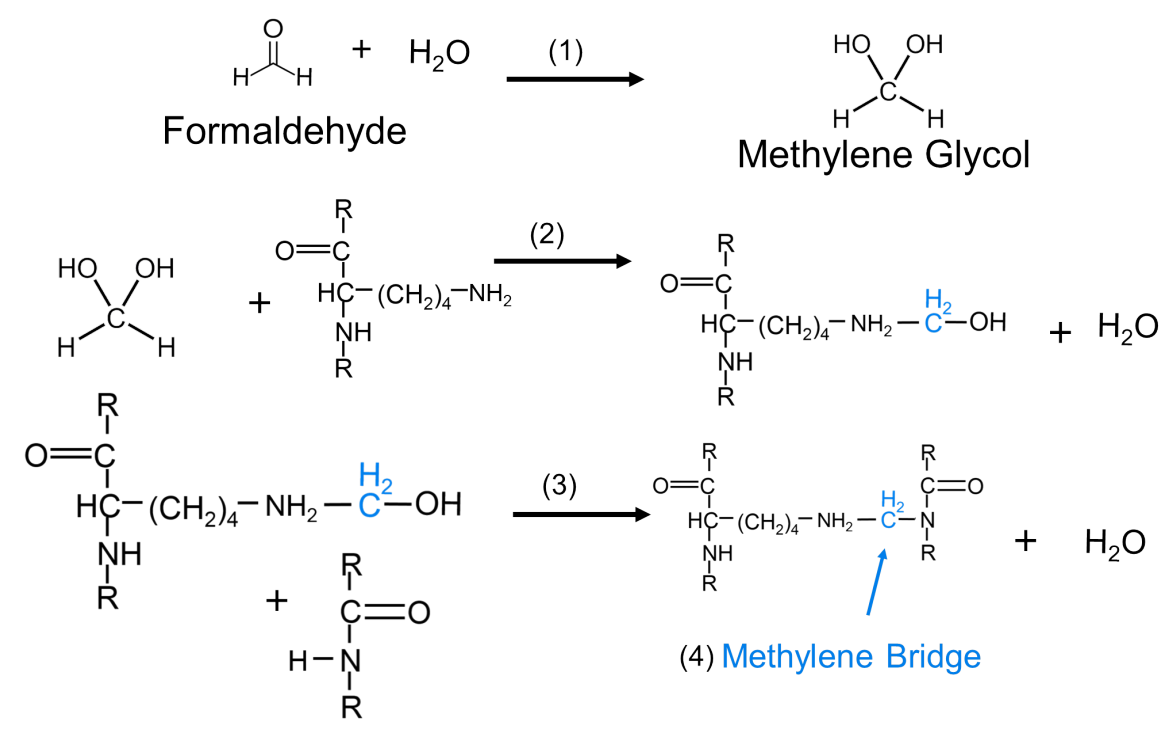

Figure 1. Formaldehyde fixation with two amino acids.

equivalent volume of medium was added to quench trypsinization and harvested cells were centrifuged at $500 \times$ $g$ for 5 min then resuspended in fresh medium for counting using a hemacytometer (Hausser Scientific, Horsham, PA) with $0.2 \%$ trypan blue (Lonza, Walkersville, MD). Passaging occurred once weekly into new flasks at a density of $1.6 \times 10^{4}$ cells $/ \mathrm{cm}^{2}$ (400,000 cells per flask) and cells were fed four days after passage by replacing $50 \%$ of the medium.

For all experiments, cells were plated and differentiated with $10 \mu \mathrm{M}$ all-trans retinoic acid (RA; Sigma, St. Louis, MS) (Clagett-Dame, McNeill et al., 2006) and allowed to differentiate for 72 hours to ensure initiation of neurite outgrowth prior to cell fixation.

\subsection{Cellular Fixation}

Cells were fixed 72 hours post-exposure with either a solution of 4\% paraformaldehyde and 1\% Triton X-100 in PHEM buffer (60 mM PIPES, 25 mM HEPES, 10 mM EGTA, 2 mM $\mathrm{MGCl}_{2}$, pH 6.9) or a solution of $8 \%$ malonic acid and 1\% Triton X-100 in PHEM buffer. Half the medium $(250 \mu \mathrm{l})$ was removed from each well and $500 \mu \mathrm{l}$ fixative solution was added. Cells were then incubated for 7 minutes at room temperature, followed by replacement of $500 \mu \mathrm{l}$ in each well with another dose of fixative, again incubating cells for $7 \mathrm{~min}$ at room temperature. By not performing complete medium removal, the fluid remaining in each well buffered the cells and their neurites against turbulence caused by addition of more liquid. Once fixed, cells are more strongly bound to their substrate and are unable to retract neurites, making them much more resistant to the mechanical disturbances necessary in the staining procedure. When fixation was complete, all liquid were removed from each well and replaced with phosphate-buffer saline (PBS). Slides were stored at $4{ }^{\circ} \mathrm{C}$ overnight and stained the following day.

\subsection{Immunocytochemistry}

For staining cytoskeletal components, fixed cells were washed and permeabilized with PBS containing $1 \%$ Triton X-100 (PBS-TX) for 30 min, then blocked with 10\% normal goat serum (NGS) in PBS for 30 min. Subsequent uses of $10 \%$ NGS were also diluted in PBS. The blocking solution was removed and unconjugated monoclonal mouse anti-tubulin primary antibody (1:200, Sigma) was applied for $45 \mathrm{~min}$ in 10\% NGS. Each well was then washed once with PBS and incubated for 45 min with TRITC-conjugated donkey anti-mouse secondary antibody (1:400), again in 10\% NGS. Chambers were removed and coverslips affixed with ProLong Gold antifade reagent (Molecular Probes P36934) directly following tubulin staining. Once mounted, slides were covered and left to cure for $24 \mathrm{~h}$ at room temperature prior to viewing, as per ProLong manufacturer's recommendations. All washes and treatments used a volume of $300 \mu \mathrm{l}$ per well, and all incubations were performed at 
room temperature on a rotator at moderate speed.

Cells stained for cytoskeletal elements were imaged at $600 \times$ on an Olympus (Tokyo, Japan) IX-71 fluorescence microscope equipped with a Roper Scientific (Tucson, AZ) CoolSnap HQ CCD camera and accompanying RSImage 1.9.2 software through TRITC 41002 (microtubule). Contrast adjustments were performed with ImageJ to improve visibility of fine details in figure images (Schneider et al., 2012). Corrected Total Cell Fluorescence (CTCF) was measured as outlined by McCloy et al., taking five pints per an image (2 wells per treatment, three plates total, $n=6$ ) [10]. Data was analyzed using Microsoft Excel 2010.

\section{Results}

\subsection{Malonic Acid Immunocytochemistry Comparable to Formaldehyde}

In order to test the preservation capabilities of malonic acid compared to paraformaldehyde after immunostaining, we imaged unconjugated monoclonal mouse anti-tubulin primary antibody with TRITC-conjugated donkey anti-mouse secondary antibody 72 hours after the fixation process. We found that 72 hours after fixation, malonic acid fixation (Figure 2(b)) was comparable to that of formaldehyde (Figure 2(a)) for the staining the staining of SH-SY5Y cells for tubulin. The corrected total cell fluorescence shows a similarity in the overall fluorescence of the two groups (Figure 3$)$ with statistically insignificant differences using an ANOVA $(n=6$, $\mathrm{DF}=1, \mathrm{~F}=1.46$, $\mathrm{p}$-value $=0.2469$ ).

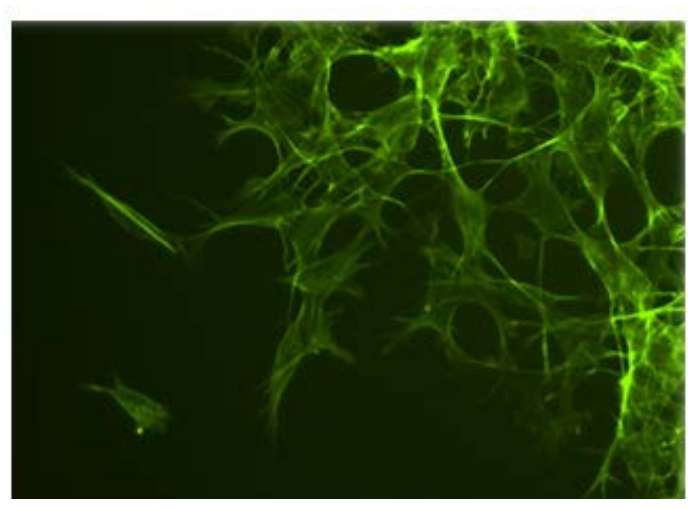

(a)

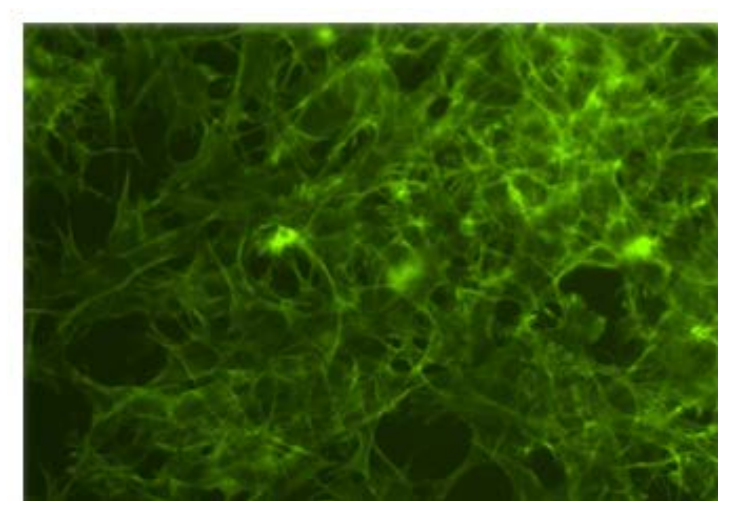

(b)

Figure 2. SH-5YSY cells fixed with formaldehyde (a) or malonic acid (b) for 72 hours.

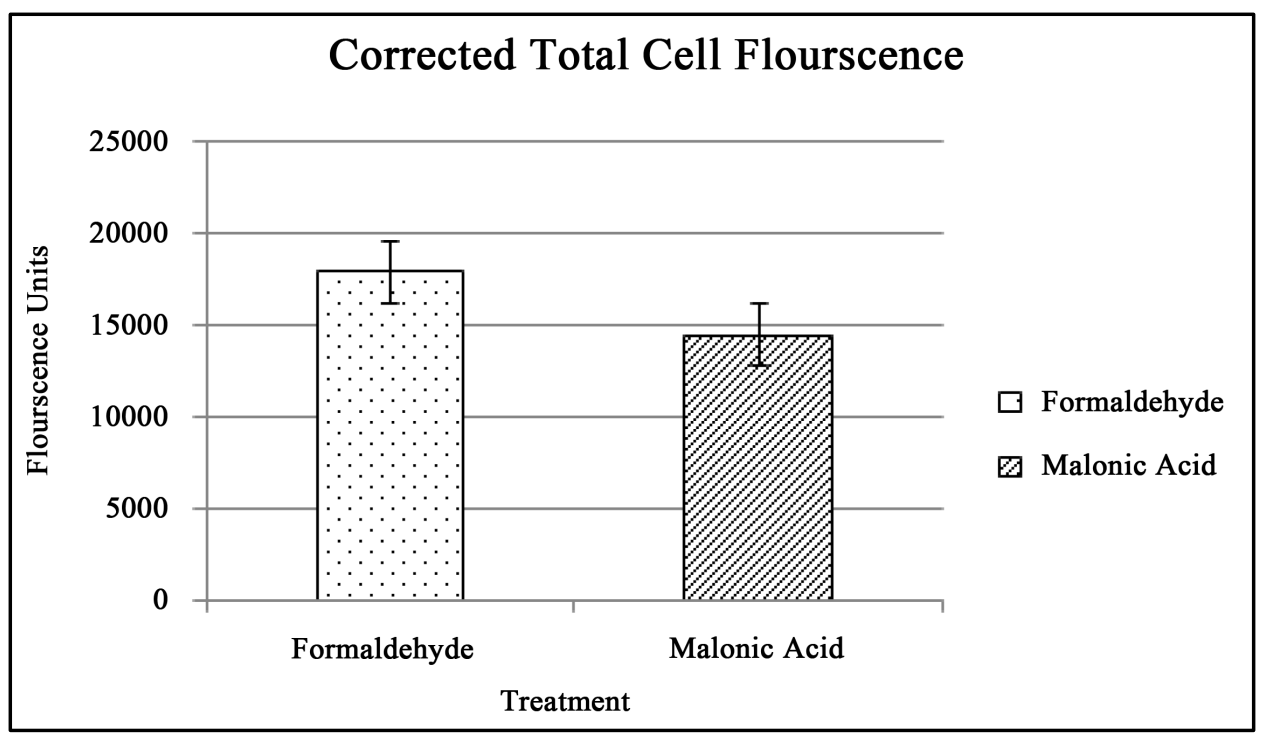

Figure 3. Corrected total cell fluorescence between formaldehyde and malonic acid after 72 hours of fixation. 


\subsection{Malonic Acid Crosslinks Proteins Similar to Canonical Mannich Reaction}

One proposed mechanism follows the Mannich reaction of formaldehyde with primary amines: 1) Malonic Acid in aqueous solution becomes hydrated to form an alcohol intermediate called 1,1,3,3-Propanetriol. 2) 1,1,3,3Propanetriol reacts with a primary amine through the nucleophilic addition of an amine to the carbonyl by dehydration to the Schiff base. The Schiff base is an electrophile which reacts in the second step in an electrophilic addition with a compound containing an acidic proton. 3) Since the solution is in aqueous solution, the hydroxyl group attached to the readily leaves due to the electronegativity of the amine nitrogen and the hydroxyl oxygen. 4) The subsequent crosslinking occurs in a similar fashion to step 2 forming a 1, Propanol Bridge. 5) The hydroxyl group on the propanol bridge readily leaves (as described in step 3). The final product is a crosslink of $-\left(\mathrm{CH}_{2}\right)_{3}$ - known as a propyl bridge (Figure 4).

\section{Discussion}

The use of formaldehyde for biological applications has been around for over a century. It is still the gold standard for cell fixation in immunocytochemical assays. Though it is widely used, it is a known carcinogen and can have severe detrimental effects on human health. Here, we show that malonic acid is a comparable alternative to formaldehyde when testing unconjugated monoclonal mouse anti-tubulin primary antibody and TRITC-conjugated donkey anti-mouse secondary antibody against formaldehyde and malonic acid fixation (Figure 1 \& Figure 3).

We showed one possible mechanism for the crosslinking of the proteins with Mannich-like fixation reaction. The use of the Mannich reaction to crosslink proteins is the canonical pathway for formaldehyde protein crosslinking and was thus an obvious choice as a mechanism for crosslinking by malonic acid, given the similarities in their electronic structure. Though our theoretical mechanism marks several differences in mechanism compared to formaldehyde (loss of water), we believe a common hydrocarbon bridge formation is the cause for the protein fixation. The mechanism shown does not provide a fully balanced equation due to the aqeous environment of the reaction. Three other proposed mechanism not explored in this paper include the use of an AzaMichael Addition to crosslink proteins, the reaction of malonic acid with cysteine thiol groups to form a propylene bridge, and crosslinking via polar uncharged amino-acids. Though we have outlined one theoretical mechanism, the exact mechanism by which malonic acid crosslinks with proteins is still open for discussion and warrants further investigation.

In addition, the crosslinking of formaldehyde has been shown to decrease the fluorescent capabilities of GFP
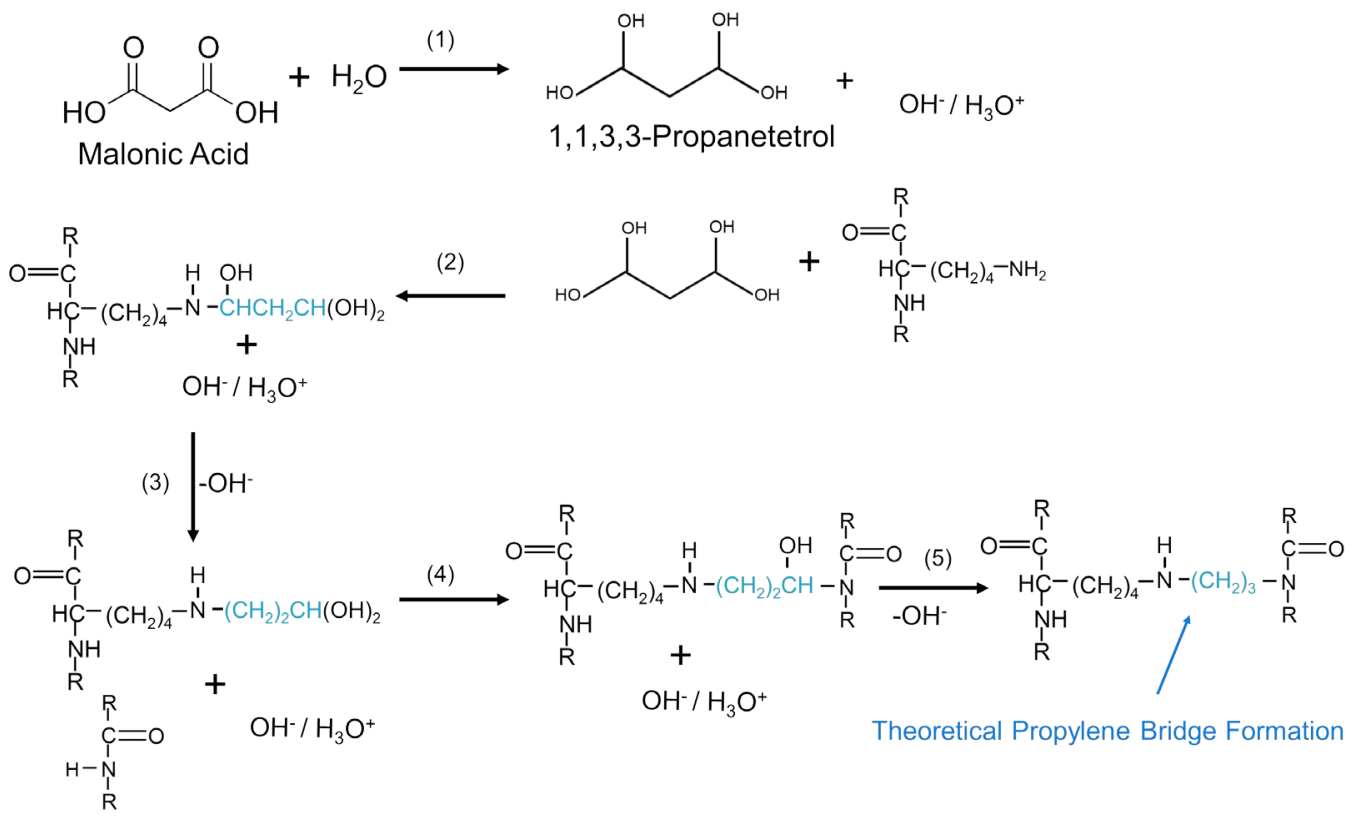

Figure 4. Theoretical malonic acid protein-crosslinking reaction. 
expressed in proteins by stabilizing the conformation of its beta barrel structure. Dicarboxylic acids such as malonic acid, in combination with other larger dicarboxylic acids could be used to ease the burden and increase the flexibility of these barrel-like structures and allow for a higher fluorescent intensity while still preserving tissue.

\section{Conclusion}

Malonic acid can be used as a greener alternative to fixing cells in vitro for immunocytochemical imaging and has comparable fluorescence output to that of paraformaldehyde, a commonly used but more carcinogenic fixative. Though we observed similar fluorescence and fixation with malonic acid, future research is required to better understand the mechanism by which malonic acid crosslinks proteins.

\section{References}

[1] Blum, F. (1893) Der formaldehyde als haertungsmittel. Z. Wiss. Mikrosk, 10, 314-315.

[2] Tian, B., Yang, J. and Brasier, A.R. (2012) Two-Step Cross-Linking for Analysis of Protein-Chromatin Interactions. Methods in Molecular Biology, 809, 105-120. http://dx.doi.org/10.1007/978-1-61779-376-9_7

[3] Blum, F. (1894) Notizüber die Anwendung des Formaldehyds (Formol) als Härtungs-und Konservierungsmittel. Anatomischer Anzeiger, 9, 229-231.

[4] Thavarajah, R., Mudimbaimannar, V.K., Elizabeth, J., Rao, U.K. and Ranganathan, K. (2012) Chemical and Physical Basics of Routine Formaldehyde Fixation. Journal of Oral and Maxillofacial Pathology, 16, 400-405. http://dx.doi.org/10.4103/0973-029X.102496

[5] Gustavson, K.H. (1956) Chemistry of Tanning Processes. http://agris.fao.org/agris-search/search.do?recordID=US201300341594

[6] National Toxicology Program (2011) NTP 12th Report on Carcinogens. Report on Carcinogens, 12, iii-499.

[7] Facke, T., Subramanian, R., Dvorchak, M. and Feng, S. (2004) Diethylmalonate Blocked Isocyanate as Crosslinkers for Low Temperature Cure Powder Coatings. Proceedings of 31st International Waterborene, High-Solids and Powder Coating Symposium, New Orleans.

[8] Dessaignes, V. (1858) Note sur unacideobtenu par l’oxydation de l'acidemalique. Comptes Rendus Geoscience, 47, 7679.

[9] Mitra, T., Sailakshmi, G., Gnanamani, A. and Mandal, A.B. (2012) Preparation and Characterization of Malonic Acid Cross-Linked Chitosan and Collagen 3D Scaffolds: An Approach on Non-Covalent Interactions. Journal of Materials Science: Materials in Medicine, 23, 1309-1321. http://dx.doi.org/10.1007/s10856-012-4586-6

[10] McCloy, R.A., Rogers, S., Caldon, C.E., Lorca, T., Castro, A. and Burgess, A. (2014) Partial Inhibition of Cdk1 in G 2 Phase Overrides the SAC and Decouples Mitotic Events. Cell Cycle, 13, 1400-1412. http://dx.doi.org/10.4161/cc.28401 